\title{
Research on plaintiff qualification of the environmental civil public interest litigation
}

\author{
Tingting SHI ${ }^{1, a}$, Yi CHEN ${ }^{1, b,{ }^{,}}$, Ting $\mathrm{ZHANG}^{1, \mathrm{c}}$ and Hui ZHONG ${ }^{2, \mathrm{~d}}$ \\ ${ }^{1}$ Tianjin Research Institute for Water Transport Engineering, M.O.T., Tianjin, China \\ 2 Shandong Taishijian Law Firm, Jinan, Shandong, China \\ a10761135@qq.com, b923418861@qq.com, c939433154@qq.com, dhuizhong1013@163.com \\ ${ }^{*}$ Corresponding author: Yi CHEN
}

Keywords: environmental civil public interest litigation, plaintiff qualification, social organization, government environmental protection department, citizen.

\begin{abstract}
With the rapid development of China's economy, environmental pollutions become severe problems that harming the social public interests. Environmental civil public interest litigation is a powerful weapon to fight pollutions and protect public interests. The plaintiff qualification of environmental civil public interest litigation directly affects the litigation process. However, there are major differences about the eligible subjects of the plaintiff in the environmental civil public interest litigation. Confirming the plaintiff qualifications of government environmental protection departments, eligible social organizations and citizens in environmental civil public interest litigation, and excluding the rights of procuratorial organs to initiate environmental civil public interest litigation, benefit the functioning of environmental public interest litigation system.
\end{abstract}

\section{环境民事公益诉讼中原告主体适格性研究}

\author{
石婷婷 $1, \mathrm{a}$, 陈怡 $1, \mathrm{~b},{ }^{*}$, 张婷 $1, \mathrm{c}$, , 钟辉 $2, \mathrm{~d}$ \\ 1交通运输部天津水运工程科学研究所, 天津，中国 \\ 2山东太史简律师事务所，济南，山东，中国
}

a10761135@qq.com, b923418861@qq.com, c939433154@qq.com, dhuizhong1013@163.com

*通讯作者：陈怡

关键词：环境民事公益诉讼; 原告资格; 社会组织; 政府环保部门; 公民

中文摘要. 随着我国经济的高速发展，环境污染问题日益严重，很多污染事件损害了社会公 共利益。环境民事公益诉讼是惩戒环境污染行为、保护环境公共利益的重要武器, 而原告主 体适格性问题又直接关系到该类诉讼是否能顺利开展。对于环境民事公益诉讼中原告的适格 主体，在理论界和实践中均有较大分歧。确认政府环保部门、符合条件的社会组织以及公民 在环境民事公益诉讼中的原告资格，并且排除检察机关发起环境民事公益诉讼的权利，有助 于环境民事公益诉讼制度充分发挥其功能。 


\section{1. 引言}

改革开放后, 我国经济尤其是工业经历了突飞猛进的几十年的发展，但伴随而来的是环 境的严重污染，其中很多污染事件严重损害了社会公共利益。环境民事公益诉讼作为惩戒污 染环境、破坏生态的行为及维护社会公共利益的有力屏障，在近几年，尤其是2012年修订的

《民事诉讼法》公布后引起了社会各界的极大关注。在环境民事公益诉讼中, 原告主体适格 性的认定直接关系到诉讼能否被法院受理而得以开展, 可以说原告主体适格性是环境民事公 益诉讼的基石。而在我国的学术界和实践中，对环境民事公益诉讼中原告主体适格性的认定 尚存有较大分歧，基于此，本文将对我国环境民事公益诉讼中原告主体适格性的问题做一些 探讨。

\section{2. 研究背景}

\section{1 环境民事公益诉讼的概念及特征}

公益诉讼这一概念起源于古罗马, 与之相对应的是私益诉讼, 从字面上理解, 基于保护 公共利益而发起的诉讼即为公益诉讼, 环境污染、食品药品安全领域等侵害不特定多数人利 益引起的诉讼就属于较为典型的公益诉讼, 而环境民事公益诉讼即为有关环境保护方面发起 的民事方面的公益诉讼。

环境民事公益诉讼的特征主要包括以下几个方面：1.诉讼原告主体的法定性，理论上， 环境民事公益诉讼中原告的适格主体必须在相关法律中有明确的规定，不符合规定的主体发 起此类诉讼, 法院一般不予受理。2.诉讼目的的公益性, 一般来讲, 发起环境民事公益诉讼 是为了维护社会公共环境利益, 这区别于私益诉讼。3.诉讼对象的特殊性，环境民事公益诉 讼以污染环境、破坏生态的行为作为诉讼对象。4.诉讼性质的特定化, 环境民事公益诉讼本 质上是民事诉讼，按照民事诉讼法的程序要求被告承担民事责任，这区别于环境行政公益诉 讼。

\section{2 环境民事公益诉讼原告主体适格性的理论基础}

有关环境公益诉讼原告主体适格性问题, 最具代表性的应该是环境权理论。环境权理论 是伴随着上世纪中期西方环境保护运动出现的，1972年在瑞典斯德哥尔摩举办的联合国人类 环境会议通过了《人类环境宣言》，此宣言中提及: “人类有权在一种能够过上尊严和福利生 活的环境中，享有自由、平等和充足的生活条件，并且负有保护和改善这一代人和将来世世 代代人的环境的庄严责任。”蔡守秋认为: “广义环境权泛指一切法律关系的主体都有使用、 享受其生存的自然环境条件的权利，也都有保护自然环境、防止环境污染的义务”[1]。

在传统民事诉讼理论中，“直接利害关系”是确定原告主体适格性的必备要素，例如根据 我国《民事诉讼法》的相关规定，发起诉讼的原告必须与案件有直接的利害关系。而在环境 权理论下, 发起环境民事公益诉讼的原告不再需要与案件有直接利害关系, 就如古代罗马法 的观点：“保护社会公共利益的诉讼，除法律有特别规定外，凡市民均可提起”[2]。梁慧星教 授则认为: “公益诉讼中的主体资格应当明晰，与以往普通的诉讼不同，此类诉讼的主体应当 是和被损害的利益没有传统上确立诉讼主体当中必须的直接利害关系”[3]。

研究西方发达国家及印度等国的环境民事公益诉讼制度，会发现原告资格的范围呈现不 断扩大的趋势, 并逐渐摆脱利害关系的束缚。但是, 是否所有的法律主体均能发起公益诉讼, 在学术界和实践中尚有争议。本文将分别就国家机关、社会组织、公民在环境民事公益诉讼 中的主体资格适格性进行探讨，并结合我国环境民事公益诉讼原告主体资格的立法和司法现 实，对我国相关立法及司法的完善提出建议。 
3. 不同主体作为环境民事公益诉讼原告的适格性

\section{1 国家机关作为环境民事公益诉讼原告的适格性}

通常来讲，作为原告发起环境民事公益诉讼的国家机关一般包括检察机关和政府环保部 门。

3.1 .1 检察机关作为环境民事公益诉讼原告的适格性

在我国，检察机关是国家的法律监督机关，基于检察机关身份的特殊性，对于检察机关 是否可以作为原告发起环境民事公益诉讼在理论界存在很大争议。根据部分学者的观点, 在 环境民事公益诉讼中作为原告发起诉讼时, 检察机关作为代表国家行使检察权的国家机关, 在话语权和专业性上相比其他适格的原告主体具备较大的优势，应将其确认为环境民事公益 诉讼的适格主体 ${ }^{[4,5]}$ 。而实际上，根据我国《民事诉讼法》第五十五条第二款的规定，立法机 关在制定相关条款时也确实是把检察机关作为环境公益诉讼的重要支持力量及最后屏障, 根 据上述法律规定，在没有符合规定的机关和组织或者上述机关和组织不发起诉讼的情况下， 检察机关可以发起环境民事公益诉讼。

然而笔者认为, 允许检察机关作为原告发起环境民事公益诉讼存在一定的弊端, 最主要 的就是角色重叠导致的对案件审理中公平性的影响。环境民事公益诉讼本质上还是民事诉讼， 诉讼的主体应当是平等的主体, 而检察机关作为原告参与民事诉讼, 将会出现其一方面作为 诉讼的原告要争取胜诉以维护公共的利益, 另一方面又要监督整个诉讼的解决过程的䢟尤局 面，这种身兼运动员和裁判员两个角色的情况势必一定程度上会造成诉讼中原被告地位的不 平等 ${ }^{6]}$, 使被告处于弱势地位, 从而影响诉讼结果的公平性。因此, 笔者认为, 不宜将检察 机关确认为环境民事公益诉讼中的适格主体。

3.1.2 政府环保部门作为环境民事公益诉讼原告的适格性

对于政府环保部门是否可以作为原告发起环境民事公益诉讼在理论界也存有一定的争 议, 主要的考虑是, 政府环保部门作为环境保护行政主管部门, 已经被赋予了对环境保护的 管理权及对破坏环境行为进行惩戒的执法权，如同时允许其作为原告发起民事公益诉讼，似 乎有多此一举之嫌, 且行政执法权和民事起诉权如何进行权衡, 如产生矛盾如何进行化解, 均会造成一定的混乱 ${ }^{[7]}$ 。

尽管允许政府环保部门作为环境民事公益诉讼的适格原告存在一定的不合理之处，但笔 者认为, 从环境保护、公共利益保护的角度看, 该设置是有益且必须的。政府环保部门作为 环境保护行政主管部门, 在诉公中有很大的专业优势, 大量的专业技术人员、环保数据及先 进的环保检测设备均有利于诉讼中的举证, 将政府环保部门作为环境民事公益诉讼中的原告, 有利于环境民事公益诉讼目的的实现。

\section{2 社会组织作为环境民事公益诉讼原告的适格性}

社会组织是在西方发达国家环境民事公益诉讼中重要的参与主体, 甚至可以说是中坚力 量, 纵观西方发达国家的公益诉讼案件, 正是因为大量社会组织的积极参与, 公益诉讼才得 以发挥出巨大的作用。笔者认为, 社会组织尤其是环保组织, 其成立宗旨和主要业务范围即 维护社会公共利益以及从事环境保护公益活动, 其成员多为志愿者, 有较高的环保热情, 而 且组织相对独立灵活, 受行政机关的约束较少, 与企业集团的利益纠葛极少, 组织数量较多, 可以更有效、更公正地行使诉讼权利。

\section{3 公民作为环境民事公益诉讼原告的适格性}

对于普通公民是否可以作为原告发起环境民事公益诉讼一直存有较大争议, 归纳起来争 议点主要有以下几个方面:（1）赋予公民发起民事公益诉讼的原告资格是否会因公民的大量 起诉而导致滥诉 ${ }^{[8]} ;$ （2）发起民事公益诉讼要求公民具有较为雄厚的财产垫付甚至承担较高 的诉讼、鉴定等费用, 且环境污染案件一般案情复杂、专业性强, 要求公民具有相当高的法 
律功底和相关的专业技能，赋予公民原告资格是否具有实际意义 ${ }^{[9]} ; （ 3 ）$ 若赋予公民发起环 境民事公益诉讼的资格，如何有效调节诉讼中社会公共利益和公民私人利益之间的平衡 ${ }^{[10]}$ 。

笔者认为:（1）关于赋予公民环境民事公益诉讼资格是否会引起滥诉的问题，考虑到诉 讼过程需要付出的财产和时间成本，结合环境民事公益诉讼的公益目的，公民发起环境民事 公益诉讼的投入和产出不成正比，笔者认为可以排除公民滥诉的可能性; (2) 关于公民是否 有能力发起并承担环境民事公益诉讼的问题，笔者认为，鉴于环境民事公益诉讼的复杂性、 专业性和诉讼成本的高昂，真正会发起环境民事公益诉讼的公民数量会很少，但这不是剥夺 所有公民发起环境民事公益诉讼资格的理由;（3）关于在环境民事公益诉讼中公民私人权益 和社会公共利益的平衡, 笔者以为, 通过合理的制度设计是可以进行有效平衡的, 随着社会 的进步和发展, 公民对于环境质量的要求逐渐提高, 公民的公共意识也在逐渐觉醒, 越来越 多的公民愿意无偿甚至自掏腰包去维护自己身边美丽和谐的环境, 去与破坏环境的行为做斗 争，通过确立公民在环境民事公益诉讼中的原告资格，可以充分调动公民的环保积极性，环 境公益才能得到最大程度的保障。

\section{4. 我国环境民事公益诉讼原告主体资格的立法和司法现实及完善}

\section{1 我国环境民事公益诉讼原告主体资格的立法和司法现实}

2012年通过修订《民事诉讼法》，我国首次通过立法确认了环境公益诉讼制度。根据该 法第五十五条的规定，法律规定的机关和有关组织可以对污染环境、侵害众多消费者合法权 益等损害社会公共利益的行为向人民法院发起诉讼。2014年修订的《环境保护法》第五十八 条进一步明确了发起环境民事公益诉讼的社会组织需要满足的条件。2015年，最高人民法院 颁布了《关于审理环境民事公益诉讼案件适用法律若干问题的解释》，该司法解释第二、三、 四、五条对环境民事公益诉讼中社会组织作为原告需要满足的条件作了更详细的规定。2017 年修订的《民事诉讼法》在第五十五条增加了第二款内容, 该款规定将检察机关作为环境公 益诉讼的补充起诉机关和支持起诉机关。根据上述规定, 我国有资格作为原告发起环境民事 公益诉讼的主体可归纳为以下两类：检察机关和环保部门为代表的国家机关、符合要求的社 会组织。但是，在我国，公民作为原告发起环境民事公益诉讼尚未通过立法得以确认。

在我国, 环境民事公益诉讼的司法实践要早于立法, 早在本世纪初, 便出现一些由检察 机关发起诉讼的案件。在我国的司法实践中，作为原告发起环境民事公益诉讼的主体主要为 检察机关、政府环保部门、环保组织等社会组织。

\section{2 我国环境民事公益诉讼原告主体资格相关立法和司法的完善}

\subsection{1 进一步明确可以发起环境民事公益诉讼的社会组织的范围}

笔者以为，尽管理论界和实践中对社会组织作为环境民事公益诉讼原告的适格性争议不 大，但并不是任何社会组织都可以有这个权利，应该对发起诉讼的社会组织有所限制，进一 步明确有资格发起该类诉讼的社会组织应该满足的条件。我国现行法律规定满足以下条件的 社会组织可以发起环境民事公益诉讼：（1）依法在民政部门登记;（2）专门从事环境保护 公益活动连续五年以上; (3) 无违法记录。笔者以为, 为防止在环境民事公益诉讼中诉权的 滥用, 还应对社会组织的经费来源、组织规模等方面进行限制, 提高社会组织的专业性和独 立承担民事责任的能力。

\section{2 .2 立法赋予公民发起环境民事公益诉讼的资格}

美国早在1970年颁布实施的《空气清洁法》当中就规定: “每个人都有资格发起环境民事公 益诉讼，特别是美国公民所享有的环境公益诉讼资格不受任何外部限制”。通过立法赋予公民 发起环境民事公益诉讼的资格, 才能发动最广大的人民群众参与到环境公益保护这一伟大事 业中来，才能使得环境民事公益诉讼这一制度发挥最大的功效。笔者认为，应该对《民事诉 
讼法》、《环境保护法》等相关法律进行修改，完善环境民事公益诉讼原告主体资格的相关 规定，明确将公民列为适格主体。

\section{2 .3 排除检察机关发起环境民事公益诉讼的权利}

尽管我国在《民事诉讼法》中规定检察机关可以在没有符合规定的机关和组织或者上述机 关和组织不发起诉讼的情况下发起环境民事公益诉讼，但笔者认为，检察机关作为民事诉讼 的原告可能会影响诉讼的公平，应排除其发起环境民事公益诉讼的权利，而要求其更多地发 挥法律监督及对具体案件进行适当支持的职能。

\section{5. 结语}

我国环境民事公益诉公制度建立时间较短，司法实践经验不够丰富，对于在诉讼中原告 主体资格的判定也存有较大分歧，亟需后期不断地进行完善。笔者认为，我国在完善环境民 事公益诉讼相关制度时，对于原告主体资格的确立，应本着以符合条件的社会组织为中心， 政府环保部门和公民为两翼, 检察机关为重要的监督和支持力量的原则进行, 以充分发挥环 境民事公益诉讼制度在环境保护方面的功能。

\section{References}

[1] S. Q. Cai, Preliminary exploration of environmental rights, Social Sciences in China, 1982,(03):29-39.

[2] P. W. Duff, Principles of Roman Law, The Classical Review, 1937,51(6).

[3] H. X. Liang et al., Justice Studies Initial Issue, Beijing: China University of Political Science and Law Press, 2002:361.

[4] Y. N. Yang, Interpretation of plaintiff qualification in environmental civil public interest litigation, Journal of Hubei University for Nationalities (Philosophy and Social Sciences), 2018,36(01):107-112.

[5] Y. Chen, Plaintiff qualification and limitation of procuratorial organs in environmental civil public interest litigation, Jinan: Shandong People's Publishing House, 2009:48.

[6] L. M. Zhang, The procuratorial organs should not be the plaintiff of environmental public interest litigation, Law Science, 2011(06):134-140.

[7] X. G. Wang, Why shouldn't EPA be a plaintiff in environmental public interest litigation, Environmental Protection, 2010(01):54-55.

[8] P. Xu, Improvement of the Plaintiff Qualification System for China's Civil Action of Environmental Public Interest, Journal of Harbin University, 2015,36(08):56-63.

[9] J. Pan, On Citizen Individual and Environment Authority are not Suitable for Plaintiff of Environmental Public Interest Litigation, Journal of Qiqihar University(Philosophy \& Social Science Edition), 2012(05):30-32.

[10]H. Lu and Y. J. Song, Research into law application of plaintiff qualification in the environmental civil public interest litigation: a case study of environmental protection organization lawsuit against chemical enterprises, Journal of Hohai University (Philosophy and Social Sciences), 2016,18(05):78-82. 